\title{
Magnetic resonance imaging methodology
}

\author{
Ewald Moser • Andreas Stadlbauer • \\ Christian Windischberger • Harald H. Quick • \\ Mark E. Ladd
}

Published online: 6 February 2009

(C) Springer-Verlag 2009

\section{Erratum to: Eur J Nucl Med Mol Imaging DOI 10.1007/s00259-008-0938-3}

This article contained two major errors:

1. An incorrect and incomplete version of the legend for Fig. 5 appeared. The correct legend is as follows:

2. A passage of the "Discussion and outlook" section was rendered wrongly:

Incorrect: (c) the most serious problems are to be expected in whole-body PET/MR at high field strengths. Limitations are due to inherent MR problems such as reduced homogeneity in large FOVs and dielectric losses, leading to signal losses, which would be further exacerbated by PET components that reduce SNR and/or increase susceptibility differences, (d) user-friendly 3D doctors, finally, integrated models will need to be developed and tested in order to better understand the origins of complex diseases.

Correct: (c) The most serious problems are to be expected in whole-body PET/MR at high field strengths. Limitations are due to inherent MR problems such as reduced homogeneity in large FOVs and dielectric losses,

The online version of the original article can be found at http://dx.doi. org/10.1007/s00259-008-0938-3.

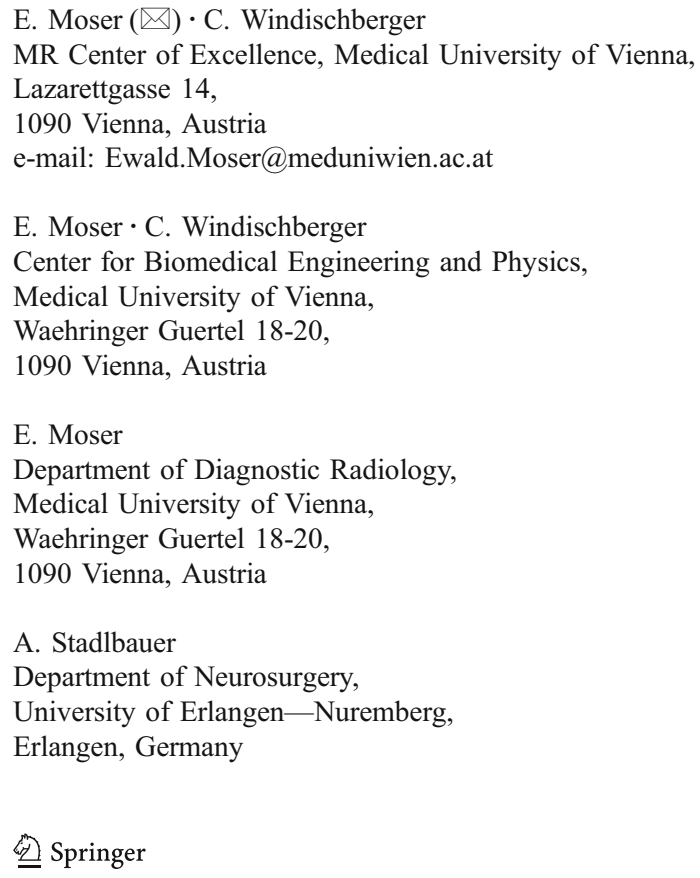

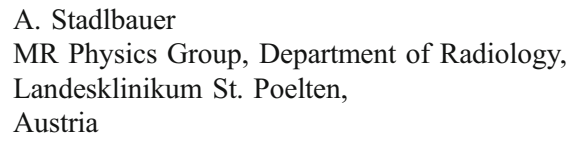


Fig. 5 Multi-modality imaging of a 39-year-old female patient with a glioblastoma multiforme IV: (a), axial T2w MR with manually segmented tumour volume (orange), pyramidal tract (blue) and volume of BOLD activation based on foot movement (green); (b) segmented map of Cho/NAA and a spectrum of the central tumour with a Cho peak; (c) coronal T1w MR fused with the volumes from (a); (d) colourcoded coronal image of fractional anisotropy (FA) as a result of a DTI experiment (blue feet-head; red left-right; green anterior-posterior); and (e) sagittal 18 F-FET PET fused with the volumes from (a). (f) Activity map from BOLD-fMRI based on foot movement
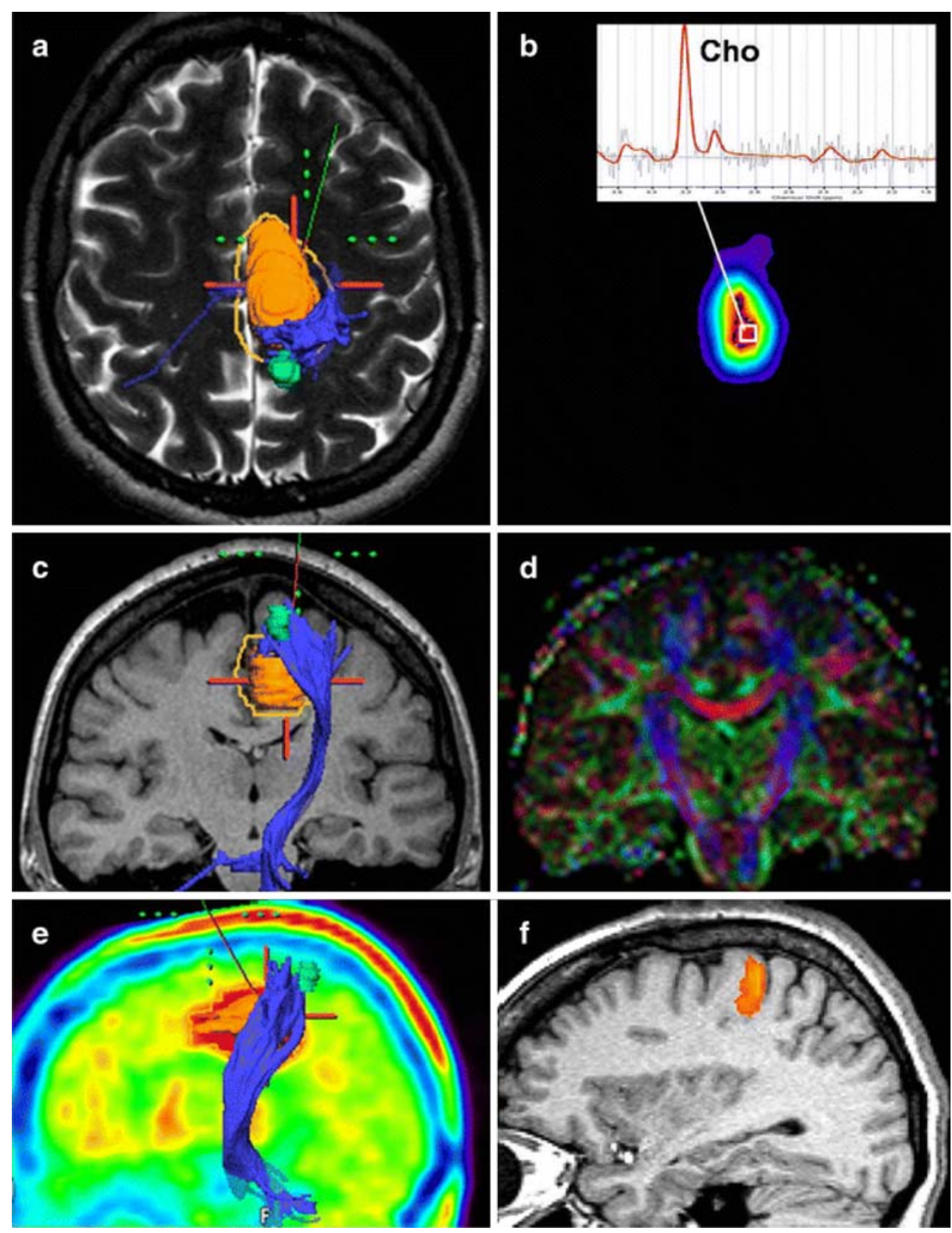

leading to signal losses, which would be further exacerbated by PET components that reduce SNR and/or increase susceptibility differences. Furthermore, user-friendly 3D multi-modality correction, fusion, and visualisation tools are required to efficiently present the large amount of information to doctors. Finally, complex models will have to be developed and tested in order to better understand the origins of complex diseases. 